\title{
EMI-RNN - Enhanced Multilayer Independently Recurrent Neural Networks for Handover Optimization in 5G Ultra Dense Networks
}

\author{
Dr K Madhavi \\ Associate Professor in CSE \\ JNTUA College of Engineering Ananthapuramu \\ kasamadhavi.cse@jntua.ac.in, +91-9440206501.
}

\begin{abstract}
With the rapid developments in the wireless communication technology, ultra dense networks (UDN) are identified as the cutting edge platform of research, in order to achieve the network capacity goals of 5G-fifth generation cellular networks. It is envisioned that the deployment of UDN plays a vital role in the development of $5 \mathrm{G}$ cellular networks to satisfy the data rate demands from the users. In an UDN, the inevitable deployment of low power base stations enables the users with many problems in terms of Quality of experience (QoE), Quality of Service (QoS) along with the major problem of frequent handovers due to the mobility of users. Addressing this problem of handover optimization in the deployment of UDN's this paper proposes a novel framework EMI-RNN based on the recurrent neural networks to predict the handovers in advance and avoid the call drop rate. Simulation results presented in the study exhibits the performance and prediction rate of the proposed framework.
\end{abstract}

Keywords: 5G Network, Ultra Dense Networks, Recurrent Neural Networks, Handovers, Handoffs

\section{INTRODUCTION}

With the drastic increase in the consumption of the bandwidth because of the current generation technological applications like smart high definition television (HDTV), voice over IP (VoIP), usage of IoT devices and graphics embedded online gaming have stimulated the progression of mobile communication networks to the 5G (fifth Generation) Ultra dense networks [1]. It is envisioned that the $5 \mathrm{G}$ UDN's are expected to deliver 1000-fold capacity increased data rates with high end mobility for the users when compared with the 4G network deployments. In specific, the ultra dense networks includes numerous small cells that enhances the rate of throughput to the static users compared with the conventional networks, simultaneously it enables with the large number of challenging issues addressing the users mobility [2]. In the context of the ultra dense networks the User Device (UD) very frequently cross the cells due to its mobility such that it requires maintaining the connections across various base stations (BS) that enables the UD with the strongest signal strength. This phenomenon of handing off the signals is called as handover (HO) mechanism [9].

The main challenges of the ultra dense networks include frequent handover drastic decrease in the quality of the service and quality of experience in the user perspective. Contemporary hand-off mechanisms will not be able to address these challenges. In this context, several solutions are proposed addressing the problem of frequent handoff's in ultra dense $5 \mathrm{G}$ networks [10]. These frequent handovers in the network may cause the packet delay and packet loss that diminishes the user experience. Furthermore, this hand-off may cause the heavy load at the network gateway that may result in the poor performance of the network. In general scenario, mobility speed of the user may not depict a major change in the signal handover problem in ultra dense networks.

The 5G ultra-dense network can be assumed as ntire network that includes several low power base stations (BS's) with varying transmitting efficiency in terms of the femtocells and picocells that are deployed randomly in the coverage region in which the large BS is called as the macrocell as shown in Figure 1.

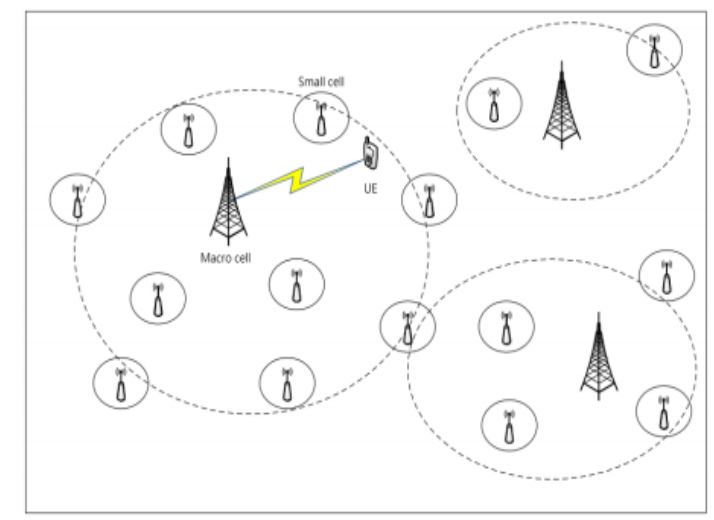

Figure1: 5G-UDN Deployment Scenarios 


\section{E-ISSN: 2321-9637 \\ Available online at www.ijrat.org}

Despite the numerous advantages in the aspect of the small cell deployment in the UDN, it includes many challenges. Handover (HO) mechanisms play a vital role of all the challenges specified in 5G-UDN [11]. The contemporary $\mathrm{HO}$ strategies adopt the process of passive trigger mechanism such that the mobile network was not made with the prior preparation that acquires an unconstructive impact on network side as well as the user side. In the user perspective, the signal interactions in between the UD, serving BS and target BS may face severe network delay and reduction in the throughput [12]. Due to the data traffic and the load at the serving BS it will not be able to accommodate the handover request from the new UD request. Therefore it is considered as a critical aspect in designing mobility management and handover mechanisms in dense networks[13]. The contribution of this study involves in designing of a novel and enhanced multilayer independent recurrent Neural Network (EMI-RNN) to optimize frequent handovers and call drop rate.

The organization of the paper is as follows section 2 presents a brief outline of the various research studies that intends to solve the problem of frequent handoffs and call drop rate using heuristic machine learning and decision making algorithms. Section 3 includes the proposed network model for the purpose of implementing the proposed algorithm. Section 4 includes the proposed framework along with its implementation scenario. Section 5 presents the simulation results. The rest of the paper includes conclusion and references.

\section{RELATED WORK}

Several studies form the researchers provided many solutions addressing the problem of handover in the wireless networks such that this section provides the glimpse of the research works and their patterns in solving the handover problem. Most of the research studies addressed the time delay and its mitigation in the context of the hand over process [ 20, 17]. Furthermore many studies indicate that there are few cases in which the handover process is too early or too late and there is a decision problem in choosing the right BS. Research studies in [18] indicate that the frequent handover problem can be solved by the implementation of the software defined networking $(\mathrm{SDN})$ in which localization of the mobility is considered as the major aspect. The studies in [19] illustrates a novel mechanism in which the small cell deployments play a major role in the context of the backhaul topology in which the researchers further concentrated on the analysis of the series of network evaluation metrics. The research work presented in [song] utilize the information theory that quantitatively evaluate the trajectory of the user device mobility such that they illustrated that the user device mobility is $93 \%$ predictable on computing the conditional entropy of the mobility history of a user device such that learning the user mobility through the trajectory data [21].

\section{A. Handover Prediction Based on ML}

Recent studies specify that the usage of machine learning has derived prolific solutions that emphasize 5G UDN's to solve various problems automatically. Figure 2 specify various machine learning algorithms that are helpful in managing handoffs in mobile networks. The most efficient and popular algorithms of those are mixtures of Gaussians, k-means, automated relevance determination (AMD) etc. These algorithms play a vital role in improvising the handover management, load balancing at the base stations, mitigation of the congestion. In this context, the handoff forecasting models utilize the dynamic handover data collected from the various base stations in which it makes use of the time series forecasting techniques as the most viable mechanism that analyze the historical patterns of the $\mathrm{HO}$ data to predict the next base station[22]. Furthermore variants of Machine learning (ML) and Deep Learning (DL) algorithms, dynamic models like Neural networks are utilized for the predictions.

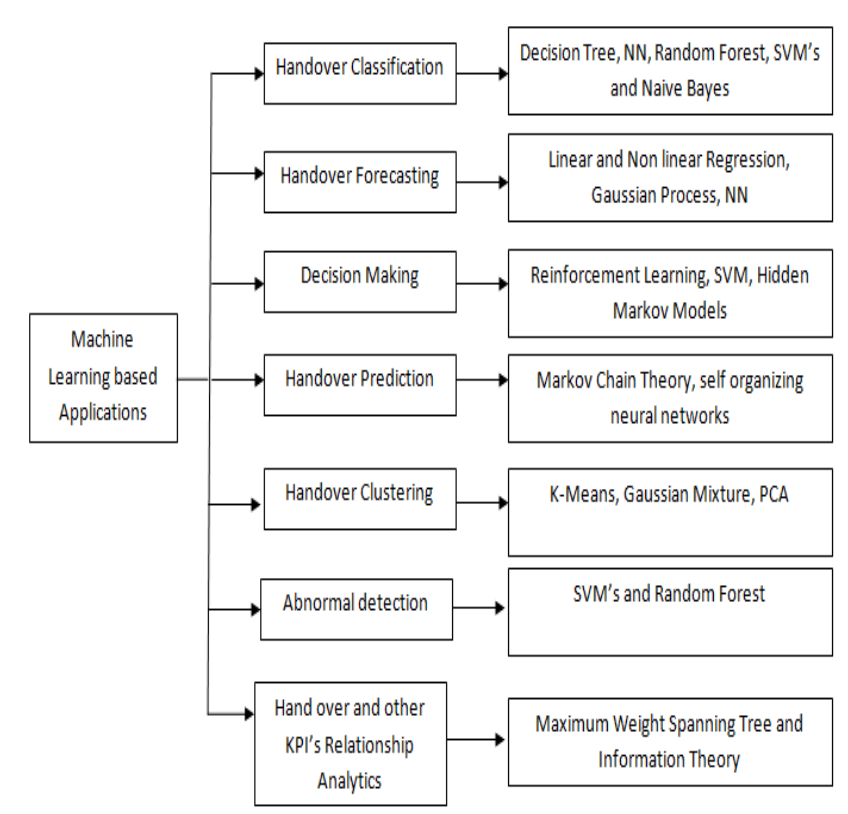

Figure 2: Variants of Machine Learning Algorithms used for Handover

Research studies have categorized the process of handovers as the Vertical HO as well as the Horizontal HO. In horizontal HO the mobility and the signal switching of the users will be happened in between the base stations (BS) of the same network 
where as in vertical handover the signal switching may happened in between the different networks. Optimizing the $\mathrm{HO}$ parameters plays a vital role as it affects the overall network coverage, energy consumption and inference management over the network. On considering its vitality progressive research is being done and several ML and DL based automated approaches are being utilized for the process.

Soldani et al., in his research studies [23], illustrated a generic framework that work based on the self optimizing networks is demonstrated as a feasible solution to optimize the Handover parameters in the context of neural networks. Supporting the feasibility of implementing the neural networks the authors in [4] have proposed a novel handoff algorithm developed based on the probabilistic neural networks and evaluated its performance based on the comparison with current generation hysteresis mechanism. It has been notified that the application of the neural network in optimizing the handoff have depicted a progressive improvement in the context of reducing overall signalling cost. Authors in [3] have brought into discussion regarding the impact changes in TTT (Time to Trigger) parameters as well as the A3-offset parameters[24]. A novel solution addressing the application of cell range extension and mobility estimation was proposed that depicts the positive impact in optimizing the Mobility Robustness of the user furthermore enhancing the performance of the network handover in an heterogeneous network distribution scenario.

Stoyanova et al., in his work [25] proposed two efficient algorithms addressing the vertical handoff optimization problem, of those the initial one is the self organising mapping (SOM) algorithm within which the handover parameters are periodically computed independently that may cause handover initiation. Furthermore the Fuzzy Logic Controller (FLC) based optimization and decision making solution that involves in the crucial metrics like latency, bit error rate, signal strength in order to determine the handover decision for each mobile terminal. The simulation results of these algorithms depict that FLC based algorithm outperforms the SOM based algorithms in optimizing and decision making in predicting appropriate handoffs. To optimize and to obtain balance in between the handoff and call drop rate, Sinclair[24] have proposed a novel mechanism that address the most vital handover parameters that include TTT and hysteresis. The proposed model is based on SOM and XSOM that enables the kernel to modify the distance metric of SOM thoroughly by allowing the mapping of the inputs within the high dimensional space. The simulation results of this algorithm depicts that it reduces the number of handoffs and call drop rate by nearly $70 \%$.

Recent class of the progressive research on frequent handoffs and call drop rate in ultra dense networks mostly make use of feedback controllers. These classes of algorithms mainly focus on the handoff parameters that include Handoff margins, TTT, A3offset and hysteresis. Wide range of research articles mainly concentrate on the Fuzzy logic controllers to solve frequent handoff problem.[6][7][8]. The pattern of research in all the research work is carried in such a way that quantifiable number if the network metrics are gathered and fuzzyfy them to make optimal decisions. On the other side Mwanje et al., in his research work [26, 5] have proposed a diverse Qlearning mechanism for mobility robust optimization in the context of addressing the handoff settings. Based on the mobility rate observed in each cell the algorithm functions and obtain penalty or reward. Authors in this research article have considered both the problems that include mobility robust optimization as well as mobility load balancing (MLB) case studies. Within the MLB the main objective is to redistribute the signalling loads automatically.

\section{SYSTEM MODEL}

\section{A. Network Model}

A simplified schematic architecture of the proposed multi-connectivity paradigm in the $5 \mathrm{G}$ ultra dense network is depicted in Figure 3.The architecture includes the trajectory movement path of the user equipment across various $\mathrm{mm}$ Wave base stations that are simultaneously connected to the LTE base stations that contain control plane connection as well as the user plane connection. In this context the user plane messages are transmitted by the LTE BS furthermore each user equipment will have a single connection which is monitored by the (MME) Mobility management equipment that which simultaneously connects the LTE BS and the core network. However the mmWave base stations can exchange the configuration messages with LTE BS through X2 link that may be wireless backhaul or wired. 


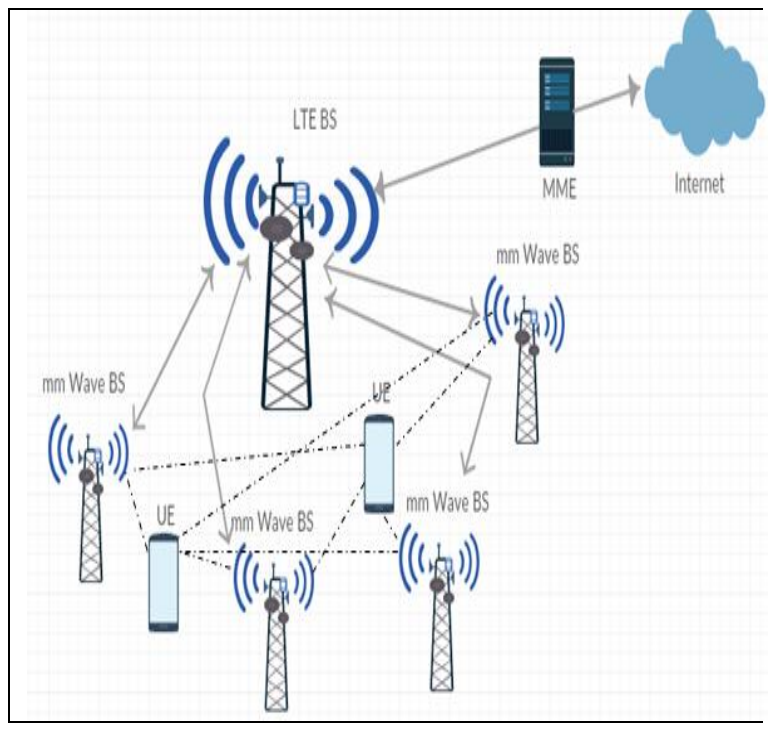

Figure 3: A simplified architecture for multiconnectivity 5G UDN

In specific, the trajectory paths of the user equipment are trained in prior based on the history of the user geographical position data. If user equipment (UE) is under service, the core network will be able to predict the further position of mobile UE at a particular moment of time based on the historical records [14]. The training and prediction of the of the network in this research work is accomplished based on the Independent Recurrent Neural Network (IRNN) phenomenon [15] derived based on the recurrent neural networks and reinforcement learning that will be explained detail in next section. The concrete process of the proposed multi-connectivity handover process is described in Figure 4.

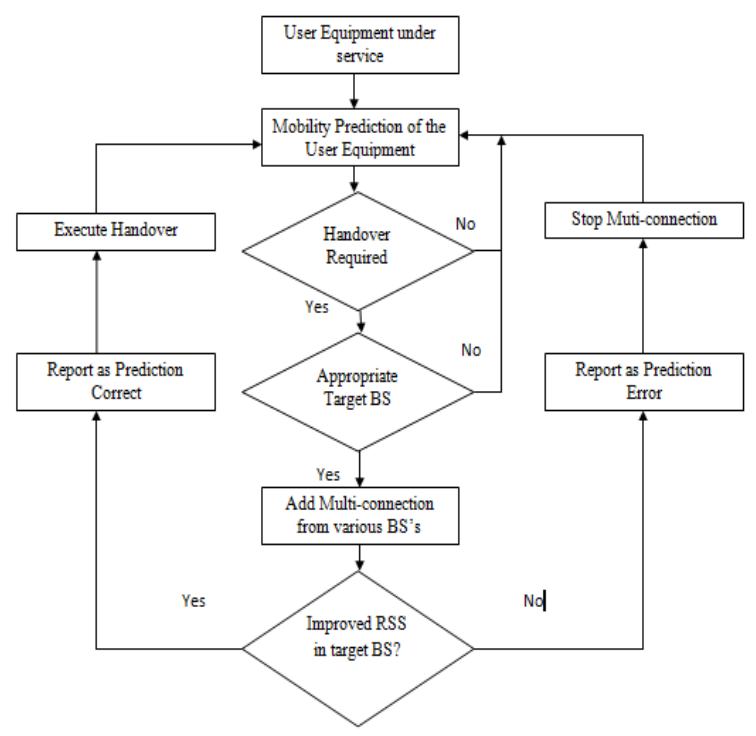

Figure 4: Handover Scenario in Proposed multiconnectivity network model
MME plays a vital role in the evaluation of future Received Signal Strength (RSS) of mobile UE and determine the next base station to optimize the frequent handoff and call drop rate. Due to the random variants in the paths of the mobile UE the network predictions may not be accurate all the time [16]. In the perspective to avoid the energy loss and call drop rate the multi connected user equipment frequently monitors the RSS from its source base stations.

\section{DEEP LEARNING OF THE NETWORK WITH EMI-RNN.}

In the context of the deep learning framework, RNN (Recurrent Neural Network) plays a major role in time series prediction. Conversely, the traditional RNN will not be able to make accurate predictions when there is a necessity to store and retrieve long term memories. Furthermore the training of the RNN experiences exploding problem as well as the gradient vanishing because of frequent multiplication of the recurrent weighted matrix. Different variants of the RNN were proposed in various research studies that include gated recurrent unit (GRU) and (LSTM) Long short term memory to address the gradient problems. To overcome these deficiencies independent recurrent neural networks are adopted as they can maintain long term memory sequences that gives more advantages over conventional RNN techniques.

\section{A. Independently Recurrent Neural Network with Backpropagation}

Independently recurrent networks are described as follows in the context of time series prediction within the networks on the utilization of the long term memory along with exploding problems and gradient vanishing

$h_{k}=\sigma\left(W t\left(X_{k}\right)+U^{\circ}\left(h_{k}-1\right)+B\right) \ldots \ldots .$. Eqn.1

Where $h_{k}$ indicates hidden vector, $W t\left(X_{k}\right)$ weight of the input vector, $U$ and $B$ indicates the matrix parameters and ${ }^{\circ}$ indicates the hadamard product. In this variant of recurrent neural network, every neuron is independent of each layer such that staking of one or more layers enhances the connection between the neurons till the $\mathrm{n}^{\text {th }}$ layer.

$h_{n, k}=\sigma\left(W t_{n}\left(X_{k}\right)+U_{n}\left(h_{n, k}-1\right)+B_{n}\right) \ldots .$. Eqn.2

In which $W t_{n}$ and $U_{n}$ indicates the n-th row of the recurrent weight and input weight such that information is received by each neuron from its hidden state of previous time step and input. In this perspective every neuron deals with a type of spatial pattern such that is considered to be as the multilayer perceptrons in which the parameters are shared. The main property of the this kind of RNN is to maintain correlation in between the neurons which are 
exploited on stacking two or more layers such that each neuron in the subsequent layer generates an output to the neurons in the previous layer.

The major drawback of the recurrent neural networks i.e., exploding problem and gradient vanishing are addressed through backpropagation of independently recurrent neural networks. The main objective of the IRNN through backpropagation is to reduce the time step $\mathrm{T}_{\mathrm{s}}$ in $\mathrm{J}_{\mathrm{m}}$ i.e., the jacobian matrix is as follows.

$$
\begin{aligned}
& \frac{\theta \mathrm{J}_{\mathrm{m}}}{\theta h_{n, k}}=\frac{\theta \mathrm{J}_{\mathrm{m}}}{\theta h_{n, T s}} \frac{\theta h_{n, T s}}{\theta h_{n, k}}=\frac{\theta \mathrm{J}_{\mathrm{m}}}{\theta h_{n, k}} \prod_{m=t}^{T s-1} \frac{\theta h_{n, m+1}}{\theta h_{n, m}} \\
& =\frac{\theta \mathrm{J}_{\mathrm{m}}}{\theta h_{n, T s}} \prod_{m=t}^{T s-1} \sigma_{n, m+1} U_{n} \\
& =\frac{\theta \mathrm{J}_{\mathrm{m}}}{\theta h_{n, T s}} U_{n}{ }^{T s-k} \prod_{m=t}^{T s-1} \sigma_{n, m+1} \quad \ldots \ldots . . . \text { Eqn.3 }
\end{aligned}
$$

$\sigma_{n, m+1}$ in the equation 3 indicates the activation function within which the gradient values includes the scalar value $U_{n}$ such that $\operatorname{diag}\left(\sigma_{n, m+1}\right)$ indicates the jacobian matrix. The learning process of the neuron includes that whenever the recurrent weight is considered as 0 the consequent neuron only uses the information of the previous neuron such that the neurons in this recurrent neural networks store values in the different patterns and in varying lengths in the memory.

\section{B. Proposed EMI-RNN mechanism to optimize frequent handovers}

The proposed EMI-RNN (Enhanced Multilayer Independently Recurrent Neural Network) mechanism is one of the variant of the independently recurrent neural network that is capable to store long stack of time series pattern for the prediction of the next layer. The optimization of frequent handover in ultra dense networks is processed in threes steps as follows:

Step 1: Initially the received signal strength at the UE is calculated from the various base stations on acquiring the time order sequence of the spatial gradient parameters of the every candidate base station such that the weights of the hidden vectors and the weight of the input signal strength is calculated. The computation process is carried out in the following manner:

Let us assume that there is N-base stations of mmWave in which two user equipment are under service. the signal strength of each base station is calculated at the LTE BS in correspondence with the UE. Further the computation of the preference level is done based on the independent recurrent neural network. Based on the activation function the preference value (hidden Vector) is computed.

$h_{1, k}=W 1\left(X_{1, k}\right)+\operatorname{diag}\left(U_{1 n}\right) h_{1, k-1} \quad \ldots \ldots \ldots .$. Eqn. 4
Equation 4 indicates computation of the preference level for primary layer simultaneously Equation 5 indicates the computation of preference level at the secondary layer.

$h_{2, k}=W 2 \cdot h_{1, k}$ Eqn.5

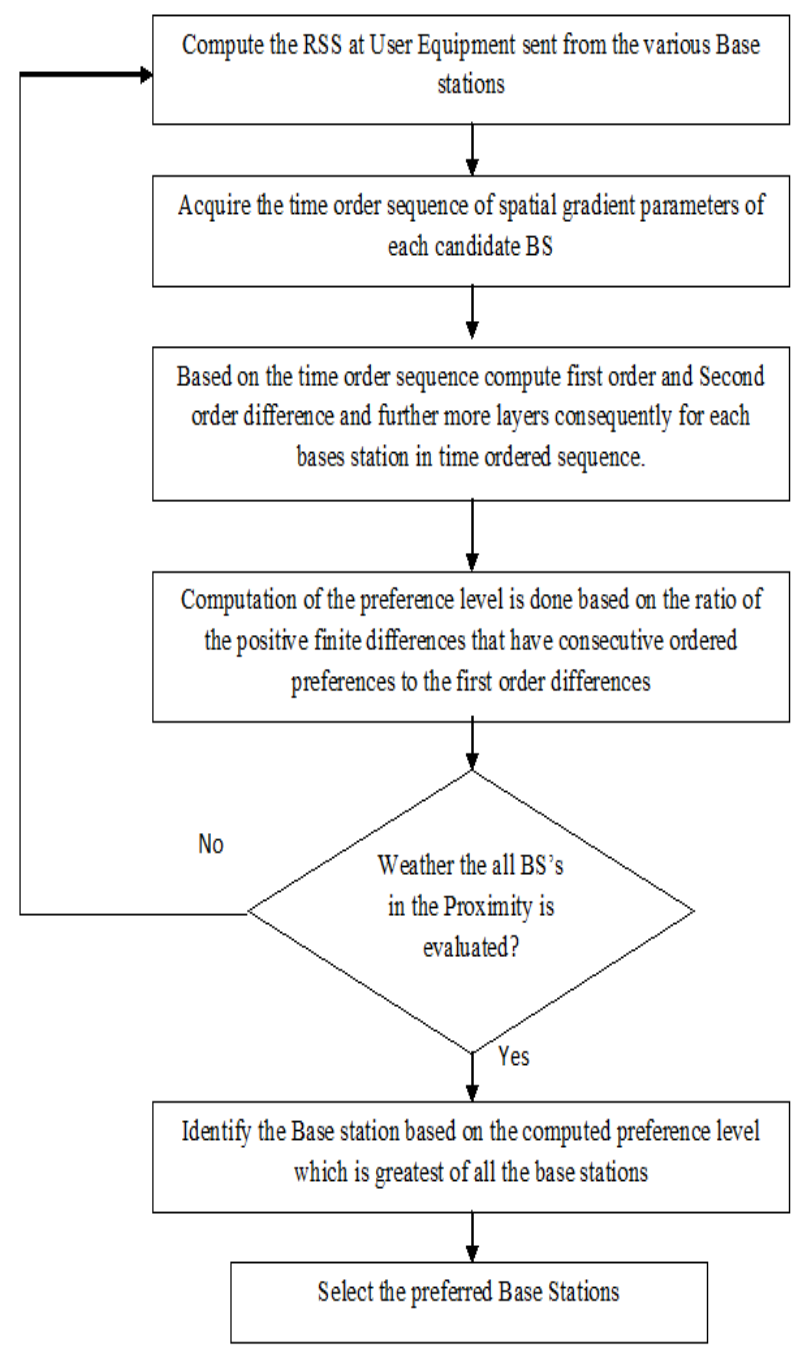

Figure 5: Flow chart of the proposed framework

Let us assume that Inverse probability of $\mathrm{W}_{2}$ is evaluated then,

$W_{2}^{-1} h_{2, k}=W 1\left(X_{1, k}\right)+\operatorname{diag}\left(U_{1 n}\right) W_{2}^{-1} h_{2, k-1}$ ..........Eqn.6 thereby,

$h_{2, k}=W 2 W 1\left(X_{1, k}\right)+W 2 \operatorname{diag}\left(U_{1 n}\right) h_{1, k-1}$ ........Eqn. 7

Finally $h_{k}=W\left(X_{k}\right)+U h_{k-1}$ Eqn. 8

Step 2: After the base station is selected for the optimized handover, the preferred base station is to be 
indicated to the LTE-BS and the mobility management equipment in which the qualitative decision is to be made in switching the base station. After obtaining the permission the UE switches to the preferred base station. Else other base station is selected quantitatively. The process of handover decision is depicted in Figure 6.

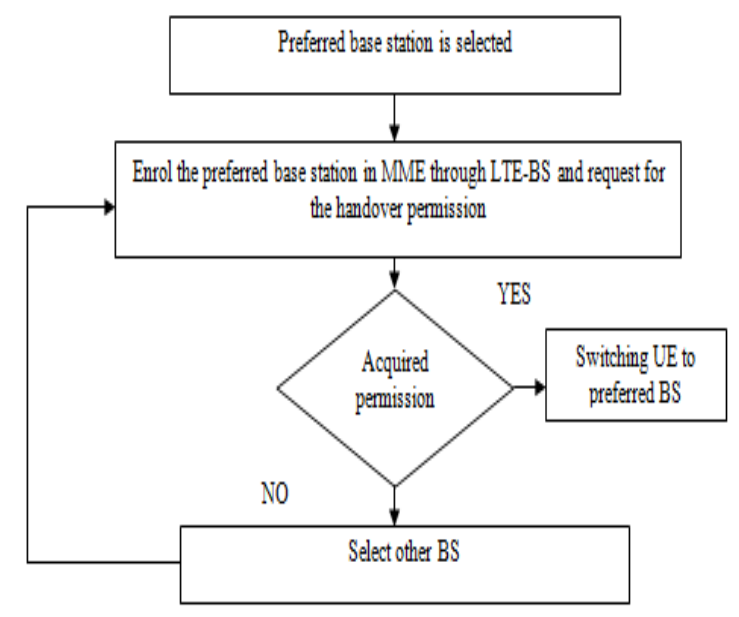

Figure 6: Handover Decision in MME

Step 3: To optimize the handover frequency the base station is selected from the list of potential base stations based on th mobility frequency of UE and the cell type of a BS. Further the process is carried on by selecting the preferred base station from the list of selected base stations.

Step 4: Repeat Step1 until the UE service is terminated.

\section{PERFORMANCE EVALUATION}

\section{A. Simulation Environment and Mobility Prediction}

Several research studies have indicated the patters of human mobility in their work that includes pausetimes, heavy-tail flights and heterogeneously circumscribed mobility areas etc. On considering these properties several mobility models were developed of those we refer (SLAW) Self-similar Least-action Human Walk data set along with the SMOOTH model in the context of generating the mobility data. Exclusive mobility patterns for the user have been generated and captured their locations for a period of 15 hours.

In this Scenario we made use of EMI-RNN algorithm to devise and exclusive prediction model for every UE in which the two dimensional coordinates of the user mobility were considered for a period of 700 minutes as an input and executed towards the time series forward a minute to obtain a standardized output. Based on that, the prediction of user mobility can be made for the next minute.

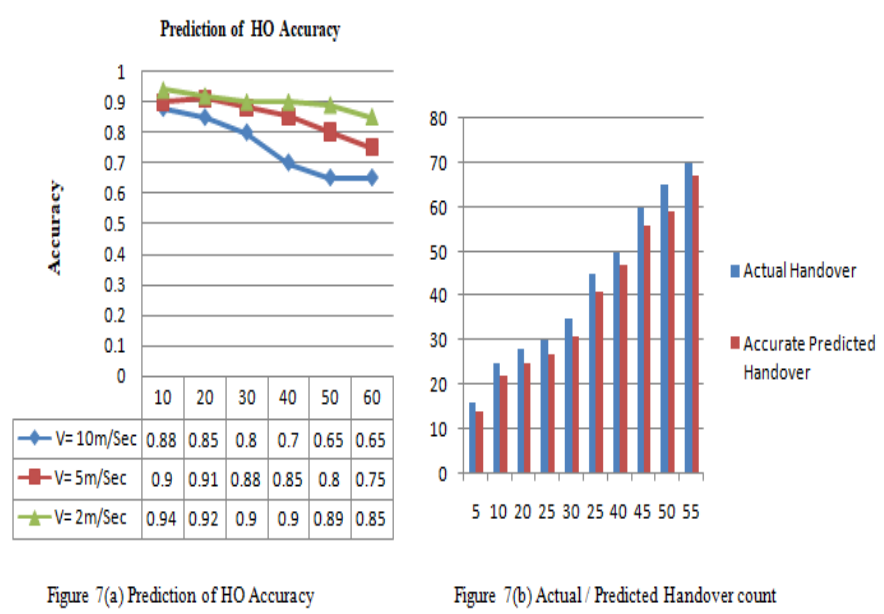

The performance evaluation of the proposed mechanism EMI-RNN is evaluated through handover prediction accuracy, moreover the comparison of $\mathrm{HO}$ prediction accuracy is assessed based on the mobility speed of the user in 3 variants such that it includes $2 \mathrm{~m} / \mathrm{s}, 5 \mathrm{~m} / \mathrm{s}, 10 \mathrm{~m} / \mathrm{s}$ respectively and compared with dual connectivity and multi-connectivity . Figure 7 . (a) Depicts the prediction accuracy of the handover and Figure 7. (b) Illustrates the frequency of the handovers based on the cell density.

\section{CONCLUSION AND FUTURE WORK}

This paper proposes a novel and intelligent framework based on multi connectivity mechanism for mobility management in 5G ultra dense networks. To learn the user equipment trajectory paths and mobility patterns this study utilizes Enhanced Multilayer Independently Recurrent Neural Network (EGI-RNN) through which a sequence of time series predictions is made possible in correlation with the mobility patterns. Based on the extensive simulations, it is identified that the predictions of Handover accuracy made by this algorithm are quite satisfied quantitatively and supports the multiple users in ultra dense networks qualitatively in terms of Quality of Service and Quality of Experience. This work can be further extended based on implementing a multiobjective decision making algorithm while delegation of the signals is being done in the low frequency signal areas. Furthermore, the proposed framework could be implemented based real time quantifiable dataset.

\section{REFERENCES}

[1] Habbal, S. I. Goudar and S. Hassan, "Contextaware radio access technology selection in $5 \mathrm{G}$ ultra dense networks," IEEE access, vol. 5, pp. 6636-6648, 2017. 
[2] A. Orsino, G. Araniti, A. Molinaro and A. Iera, "Effective RAT selection approach for $5 \mathrm{G}$ dense wireless networks," In Proc. of IEEE 81st vehicular technology conference (VTC spring), pp. 1-5, 2015.

[3] C. Cano, D. Lopez-Perez, H. Claussen and D. J. Leith, "Using LTE in unlicensed bands: potential benefits and coexistance issues," IEEE communications magazine, vol. 54, no. 12, pp. 116-123, 2016.

[4] H. Lee, H. Son and S. Lee, "Semisoft handover gain analysis over OFDMbased broadband systems," IEEE transactions on vehicular technology, vol. 58, no. 3, pp. 1443-1453, 2009.

[5] "3rd generation partnership project; technical specification group services and system aspects; telecommunications management; selforganizing networks (SON); self-healing concepts and requirements (release 11), 3GPP TS 32.541, 2012-09, v11.0.0, 2012,"

[6] “3GPP TS 36.902 evolved universal terrestrial radio access network (E-UTRAN); selfconfiguring and self-optimizing network (SON) use cases and solutions,"

[7] "3GPP TS 32.500 telecommunication management; self-organizing networks (SON); concepts and requirements,"

[8] S. Bi, R. Zhang, Z. Ding, and S. Cui, "Wireless communications in the era of big data," IEEE Communications Magazine, vol. 53, pp. 190 199, October 2015.

[9] M. Marwangi, N. Fisal, S. Yusof, R. A. Rashid, A. S. Ghafar, F. A. Saparudin, and N. Katiran, "Challenges and practical implementation of selforganizing networks in LTE/LTE-Advanced systems," in Information Technology and Multimedia (ICIM), $2011 \quad$ International Conference on, pp. 1-5, IEEE, 2011.

[10] Q. Han, S. Liang, and H. Zhang, "Mobile cloud sensing, big data, and $5 \mathrm{G}$ networks make an intelligent and smart world," IEEE Network, vol. 29, pp. 40-45, March 2015.

[11] K. Zheng, Z. Yang, K. Zhang, P. Chatzimisios, K. Yang, and W. Xiang, "Big data-driven optimization for mobile networks toward 5G," IEEE Network, vol. 30, pp. 44-51, January 2016.

[12] M. Musolesi, "Big mobile data mining: good or evil?," IEEE Internet Computing, vol. 18, no. 1, pp. 78-81, 2014.

[13] M. A. Imran, A. Imran, and O. Onireti, "Participatory sensing as an enabler for selforganisation in future cellular networks," IOP Conference Series: Materials Science and Engineering, vol. 51, no. 1, p. 012003, 2013.

[14] K. L. Mills, "A brief survey of self-organization in wireless sensor networks," Wireless Communications and Mobile Computing, vol. 7, no. 7 , pp. $823-834,2007$
[15] J. G. Andrews, S. Buzzi, W. Choi, S. V. Hanly, A. Lozano, A. C. Soong, and J. C. Zhang, "What will 5G be?" IEEE J. Select. Areas Commun., vol. 32, no. 6, pp. 1065-1082, 2014.

[16] M. Kamel, W. Hamouda, and A. Youssef, "Ultra-dense networks: A survey," IEEE Commun. Surveys Tuts., vol. 18, no. 4, pp. 25222545, 2016.

[17] S. F. Yunas, M. Valkama, and J. Niemela, "Spectral and energy efficiency " of ultra-dense networks under different deployment strategies," IEEE Commun. Mag., vol. 53, no. 1, pp. 90-100, 2015.

[18] J. Wu and P. Fan, "A survey on high mobility wireless communications: Challenges, opportunities and solutions," IEEE Access, vol. 4, pp. 450-476, 2016.

[19] C. Song, Z. Qu, N. Blumm, and A.-L. Barabasi, "Limits of predictability' in human mobility," Science, vol. 327, no. 5968, pp. 1018-1021, 2010.

[20]Z. Becvar and P. Mach, "Adaptive hysteresis margin for handover in femtocell networks," in Proc. IEEE ICWMC 2010, Valencia, Spain, Sept. 2010.

[21] H. Zhang, X. Wen, B. Wang, W. Zheng, and Y. Sun, "A novel handover mechanism between femtocell and macrocell for LTE based networks," in Proc. IEEE ICCSN 2010, Singapore, Feb. 2010.

[22] Song, Z. Qu, N. Blumm, and A.-L. Barabasi, "Limits of predictability' in human mobility," Science, vol. 327, no. 5968, pp. 1018-1021, 2010.

[23] Soldani, G. Alford, F. Parodi, and M. Kylvaja, "An autonomic framework for self-optimizing next generation mobile networks," in 2007 IEEE International Symposium on a World of Wireless, Mobile and Multimedia Networks, pp. 1-6, June 2007

[24] N. Sinclair, D. Harle, I. A. Glover, J. Irvine, and R. C. Atkinson, "An advanced SOM algorithm applied to handover management within LTE," IEEE Transactions on Vehicular Technology, vol. 62, pp. 1883-1894, Jun 2013.

[25] M. Stoyanova and P. Mahonen, "A next-move prediction algorithm for implementation of selective reservation concept in wireless networks," in 2007 IEEE 18th International Symposium on Personal, Indoor and Mobile Radio Communications, pp. 1-5, Sept 2007

[26]S. S. Mwanje, N. Zia, and A. Mitschele-Thiel, "Self-organized handover parameter configuration for LTE," in 2012 International Symposium on Wireless Communication Systems (ISWCS), pp. 26-30, Aug 2012 